of, say, a new system of income tax ? The immediate need is that governments should be forced to be more open on technological matters. The second is that the scientific community should help to guide informed and level-headed discussion. But it is significant that one of the most important influences on the direction of technological development is the old-fashioned interaction between supply and demand. Anybody who doubts that has only to ask how else than by popular acclaim has the automobile industry grown to its present size. And in this sense, of course, one of the causes of anxiety about the future of tcchnology is that it is hard to predict just what will happen next. The uncertainty may seem more acute than it used to be, but it is not intrinsically novel. The problem is to live with it. Medawar and Hobbes should help.

\section{RADIO ASTRONOMY}

\section{More Telescopes Urged}

\section{from our Astronomy Correspondent}

A CRITICAL need for new radio telescopes in the United States is urged by the National Science Foundation committee under the chairmanship of $\mathbf{R}$. H. Dicke. In the committee's second report, issued last week, the Ad Hoc Advisory Panel for Large Radio Astronomy Facilities says that the momentum of radio astronomy in the United States will be lost unless a start is made immediately on new telescopes. The members of the panel are B. J. Bok (University of Arizona), S. A. Colgate (New Mexico Institute of Mining and Technology), R. Kompfner (Bell Telephone Laboratories), W. W. Morgan (Yerkes Observatory), E. N. Parker (University of Chicago) and G. Westerhout (University of Maryland). The chief cause for dismay seems to be that the complement of radio telescopes available to United States astronomers is the same as it was two years ago, when the panel last reported, and there are no firm plans for new instruments. What the panel would like to see is a resurfacing of the dish at Arecibo so that it can be used at wavelengths of $10 \mathrm{~cm}$ or less, an array of eight dishes of 130 foot diameter at the Owens Valley Observatory of the California Institute of Technology and a 440 foot steerable dish and a large array of twenty-seven antennac at locations not yet decided. The panel also recommends design ștudies on large steerable dishes to be used at wavelengths of $3 \mathrm{~mm}$, a substantial improvement in the support for radio astronomy in the universities and at least half the observing time on large instruments to be available for visitors from elsewhere in the United States.

The report is published at a time when American ground-based astronomy is in the financial doldrums, with radio astronomy coming off if anything worse than optical astronomy. By the financial year 1970 , federal backing for radio astronomy is expected to be down to $\$ 5 \cdot 45$ million, from $\$ 7$ million in 1968 . Support for optical astronomy is also to drop over the same period, but by 10 per cent compared with 22 per cent for radio astronomy. The National Science Foundation component is an unsuccessful attempt to slow the trend, caused as much as anything by a decrease of interest in pure astronomy by NASA and by the Department of Defense. In 1970, the National Science
Foundation expects to spend $\$ 3$ million on radio astronomy, an increase of nearly \$I million on the $\$ 2 \cdot 14$ million spending in 1968. But NSF support for optical astronomy is to drop from $\$ 4.06$ million to $\$ 3.8$ million during the same interval.

Against this background, the panel's recommendations seem a far cry from what United States radio astronomers are likely to get in the near future. Little confidence is generated by the reaction to the panel's 1967 report, in which a similar series of recommendations was put forward but no new telescopes were started. The latest report was commissioned to review the 1967 recommendations, and says that the needs for new telescopes which were urgent two years ago have now become critical. In particular, the panel records its disappointment that the resurfacing of Arecibo recommended in 1967 has not been carried out. The 1967 estimate of the cost was a modest $\$ 3$ million.

The 440 foot steerable dish and the large array of twenty-seven antennae which are recommended would both be built in the south-west United States. The steerable dish is based on a design study by the Northeast Radio Observatory Corporation, and would be enclosed in a radome. In the opinion of the panel, a dish of this size is well within technical limits. By protecting the dish in a radome, a lighter and cheaper structure can be built, although radome losses mean that the instrument is equivalent to an unenclosed dish of 400 feet. In 1967 the cost would have been $\$ 28$ million spread over five years.

The large array is a proposal of the National Radio Astronomy Observatory, and would be built in sections with each section becoming operational while construction proceeded. According to the panel, it would produce up to three radio pictures per day with a resolution of $1^{\prime \prime}$ of arc-as good as optical photographs.

Both arrays of small antennae and individual large dishes are needed by radio astronomers. The arrays are used to produce high resolution radio pictures of particular objects-the large dishes complement them with low resolution sky surveys, line spectroscopy and observations at a range of wavelengths among other things.

\section{EDUCATION}

\section{Italian Policy Reviewed}

THe system of secondary education in Italy has progressed a long way since the war towards meeting increasingly complex social and economic demands, but, according to a survey by the Organization for Economic Cooperation and Development, there are still a number of areas, particularly the supply of teachers and the structure of science teaching, in which reforms are urgently needed. The report (Review of National Policies for Education-Italy, HMSO, $£ 268$ ) is part of the OECD's programme of helping member countries to evolve national science and education policies. It concentrates chiefly on secondary and particularly upper secondary education where the most rapid expansion is occurring and where the conflicting demands of general culture and specific job qualifications have to be resolved. It is based on a report by a group of examiners together with the 